\title{
Post-Simulation Visualization Application for Production Improvement of Modular Construction Manufacturing
}

\author{
M. Moghadam1 ${ }^{a}$ B. Barkokebas2 ${ }^{a}$ and M. Al-Hussein $3^{a}$ \\ ${ }^{a}$ Hole School of Construction, Department of Civil and Environmental Engineering, University of Alberta, Canada \\ E-mail: mana.moghadam@ualberta.ca,barkokeb@ualberta.ca, malhussein@ualberta.ca
}

\begin{abstract}
-
The modular construction manufacturing (MCM) process is a complex operation that combines line flow product movement with a complex activity precedence network. There are physical constraints related to the given facility, as well as logical constraints caused by demand variation. In order to change production line layout and make improvements within the context of Lean, a tool is needed to assist MCM to quantify, at the planning and evaluation stages, the benefits they can expect from proposed changes to their system. Simulation is a technique by which to facilitate identifying changes and benefits of future transformation and to determine where valuable resources should be
\end{abstract} applied prior to actual implementation.

Despite the benefits of simulation, project management teams typically are unwilling to make decisions based on current simulation outputs, since they are very difficult to understand and require specialized skills in interpretation of the information. Visualization is a more popular technique since it fosters better understanding of the construction process. However, to be effective for decision making purposes, such a model must be linked to project information. The visual interpretation of the simulation results constitutes a more effective approach. In this paper, a simulation model is thus generated to provide results for different production scenarios, and then the near-optimum scenarios are run to visualize the production constraints, which facilitates precise scenario comparison. The developed model capitalizes on the advantages of both simulation and visualization, whereby critical information such as the 3D model, time constraints, and resource demand are incorporated into the system. The proposed methodology is validated by a case study, a residential modular factory in Edmonton, Canada, which illustrates the effectiveness of the proposed methodology.

Keywords -

Modular Construction Manufacturing; Lean; Production; Simulation; Visualization; Resource Utilization

\section{Introduction}

Modular construction manufacturing (MCM) provides opportunities to apply Lean for production efficiency in the plant, thereby eliminating waste and supporting the delivery of products in a shorter time and at a lower cost. The decision to implement Lean is complicated because of the substantial differences between a traditional system and a Lean system. What is needed is a tool to assist organizations considering Lean to quantify, at the planning and evaluation stage, the benefits they can expect from applying Lean. This tool should be adaptable to the specific circumstances of the organization, and should be capable of generating resource requirements and performance statistics for both the proposed Lean system and the existing system [1]. Simulation is an effective technique to facilitate identifying changes and benefits of Lean transformation. Simulation is a computer-based tool that represents realworld objects and processes in order to effectively evaluate and examine various scenarios prior to implementation and facilitate the decision-making process [2]. The impact of Lean transformation can be analyzed to determine where valuable resources should be applied before actual implementation. This increases confidence and is likely to hasten the rate of adoption of Lean, as it provides a visual and dynamic illustration to management of how the new system would work [1].

On the other hand, to make improvements on a production line within the context of Lean, the integration of simulation tools and Lean brings about a more effective approach for process management. A simulation model of the production line stream map is able to challenge the impacts of Lean application on line balancing, as well as the results [3]. Significant impact from random variation in the nature of modular manufacturing, as well as system element interactions, is expected for the future-state of the system. Currently there is no tool in Lean which contains variability data and provides performance evaluation of the system. Therefore, incorporating simulation modeling into the Lean transformation process is able to provide a quantitative conception of the potential benefits [4]. 
Although the simulation tool is considered to be efficient in evaluating construction processes, the project management team often views it as a "black box" that can only be understood by highly skilled managers. The gap between the specialist and the management team leads to misunderstandings regarding simulation results, and may result in incorrect interpretations [5]. The project management team is unwilling to make decisions based on current simulation outputs, such as statistics-based charts and diagrams, because they do not provide adequate information related to construction process requirements [6]. The results and analysis are difficult to understand, and translation of the data must be done by experts [7]. Still, visualization is widely accepted in construction since it promotes better understanding of process and performance of the construction system. However, the model needs to be associated with the project data in order to be effective and valuable for decision making purposes. The key aspect that is added to the output analysis of the simulation process is visualization, leading to postsimulation analysis. The produced results from a simulation model are interpreted and visualized for further analysis, which is a popular and widespread decision making technique [8].

The MCM industry is a growing industry that seeks innovative approaches to increase profitability. To improve the production efficiency in the plant, an enhanced tool is required to present the statistical results of changes in production sequencing and crew selection in a visualized model to facilitate decision making. The application of visualization to construction in general differs from its application to MCM in terms of production flow of multiple projects, product movement through the production line, work cell design, precedence network lead time, plant physical constraints, and crew selection. Also, with MCM the production progress is not measured based on the physical project progress, but instead is determined based on the total work performed in all stations, and can be calculated as the sum total of man-hours. Numerous studies have investigated the integration of simulation and visualization for on-site construction, but only a few have accomplished this for modular manufacturing. Therefore, research in the area of on-site construction is being carried out to develop and advance an integrated tool for MCM production process analysis.

In this paper, the complicated result from the simulation model of the work process provided monitoring of both the visualization and the simulation parameters simultaneously by providing additional perception through visualization, and also provided the immediate visualized or statistical results of making changes to the model. Based on that, the simulation results of near optimum scenarios are run to simulate the process and visualize the production constraints. This integrated method is a post-simulation visualization model for MCM, with an emphasis on production efficiency. The model capitalizes on the advantages of both simulation and visualization, where critical information such as the 3D model, time constraints, and resource demand are incorporated into the system.

\section{Research Methodology}

The methodology process in this research is divided into five major phases. In Phase I, a simulation model of production flow is developed in order to depict the production line layout, schedule, and resource requirements. The inputs for this model consist of information extracted from a BIM-generated 3D model, resource requirements, and proposed improvements. The simulation model delivers results for different production scenarios and provides the opportunity to evaluate the proposed future-state in order to optimize the production process. In Phase II, a post-simulation visualization model of evaluated production scenarios is developed which can be used by management teams as a more efficient tool for production flow analysis. As a result, the resource requirements to complete various modules are determined, along with potential scenarios for work flow balancing.

\subsection{Phase I. Developing Simulation Model}

Simulation tools play an important role in the application of simulation in different industries. Simulation in construction had been limited to research applications before AbouRizk and Hajjar [9] presented a framework that customized simulation for the construction industry. They used Special Purpose Simulation (SPS) as a tool to build systems for specific purposes. Later, they improved their approach and developed a construction simulation system, called Simphony, which provides a set of predefined elements representing construction requirements [10]. Simphony.NET is a construction-oriented, generalpurpose discrete event simulation (DES) software application developed at the University of Alberta using process interaction concepts to create a model. A general template with predefined element functionality enables the user to select elements based on a required function for a specific simulation model. In this research, Simphony.NET 4.0 is used to develop a simulation model for the production process. The process to generate and evaluate the simulation model is presented in Figure 1. In the simulation model, the value stream map of the case-study is created to show the product 
family, information and material flow, work cells, inventory amount, daily customer demand, supplier and shipping schedule, and production volume. The generated simulation model in Simphony.NET 4.0 involves two input types: Fixed and random variable. The values of fixed variables, such as number of entities, change over time, yield rate, value-added time, and transportation time between stations, remain constant during a simulation run. These fixed inputs are defined by the user according to the factory and project specifications. The values of random variable inputs, such as process time and number of operators, change according to data distributions during the simulation run. These random variable inputs are probabilistic distributions of various activities based on the module specifications.

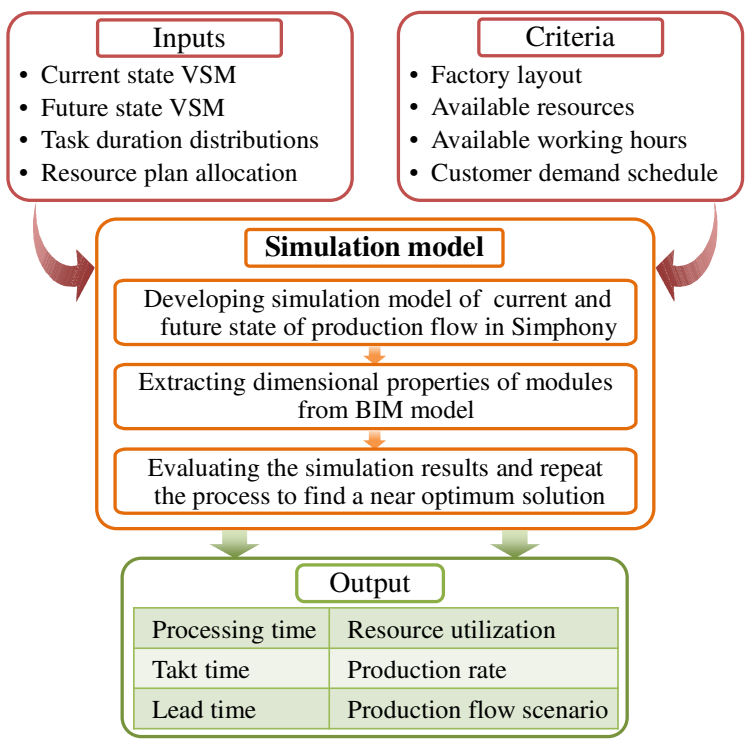

Figure 1 Process for generating and evaluating the future-state through simulation

Generated outputs from the simulation model include total processing time, lead time, Takt time, labor utilization, production rate, and potential production flow scenarios. The simulation model outputs of various scenarios are evaluated in order to find a near optimum result. The optimum result addresses the following goals: (1) increased productivity, (2) balanced labor allocation, (3) satisfied customer demand in terms of schedule, (4) reduced or eliminated waste, (5) reduced delays, (6) eliminated bottlenecks and unnecessary material handling, (7) addressed defects and problems, and (8) optimized space usage. Evaluation of potential scenarios and comparison of the results assists management teams to find optimum production scenarios prior to actual implementation.

\subsection{Phase II. Developing Post-Simulation Visualization Model}

The proposed post-simulation visualization (PSV) is the 3D visualization of the DES model, representing physical working environments with 3D graphical objects. The model depicts the simulation of the production process in detail, producing and displaying production flow information simultaneously such as lead time, production rate, and resource utilization for evaluation purposes. The proposed system has the capacity to be linked to all possible production information. The model needs to be flexible in order to deal with changes in process caused by module variation and to present a complex production process in a simple way. Visualization of the simulated process is proven to be an effective tool in communicating the value and simplicity of the minute-by-minute schedule. The simulation result comparison between the initial model before applying PSV and the final version after running PSV several times shows significant improvements in terms of eliminating waste, smoothing the production flow, leveling resources, and reducing idle time. A comparison is also conducted among various scenarios, by which the management team confirms the considerable impact of the PSV model in terms of decreasing activity durations, eliminating errors and rework, and identifying the best potential production scenarios using visualization of the simulated process during the planning phase. In this research, two approaches are performed to create a PSV model. The first approach integrates animation with statistical outputs of simulation model and provides a lifelike image of the process. The second approach is more dynamic, capable of responding instantly to changes made to the process.

In the first approach, to create a real-time abstract simulation model by means of computer animation, a visual presentation of production scenarios is developed. After evaluating and comparing results among various potential production scenarios, a scenario with near optimum results is selected for visualization. A 3D model of the production process is also developed in Autodesk 3D Studio Max (3ds Max) showing certain activities at all the stations in the production line. The processing times of all the stations are imported from the simulation model output in the form of an ASCII file, which is a binary text file, and are linked to the 3D animation semi-automatically. Within the 3D animation environment the high-level simulation model is transformed to a micro-level representation in frames/minute. To develop the PSV model, 3ds Max's scripting language, MAXScript, is used. As inputs of the PSV model, two sources are required: (1) the 3D model library of PSV; and (2) the simulation model output that stores the spatial configuration of the 
construction process, along with performance time. The PSV model imports 3D models from the 3D library, including models of the equipment, modules, resources, and the 3D factory, and assembles them in 3ds Max. Then the 3D animation engine uses the data from the simulation output file in order to create the key frames. The model's outputs are production processing time, labor utilization, safety and quality control, and evaluation of potential scenarios for construction operations.

The second approach is pursued to create a more dynamic visualized model from simulation results which can be modified by any user with no special software skills required. For this purpose Autodesk Navisworks Manage is selected, which combines clash finding analysis and interface management with 4D schedule simulation. The 3D model of the factory is developed in Revit and then, along with 3D models of modules generated in the design phase, is imported into Navisworks. The imported outputs from the simulation model for existing modules in the production line and coming modules to be visualized include the processing times at all stations, resource allocation plan, and work sequence. In order to analyze different scenarios for plant layout configuration with automatic clash detection, more information is added to the model, including overhead crane capacity, factory space limitations, and labor safe work area. In this model, the relationships among components are defined through parametric modeling rules and constraints, such that the model responds automatically to any changes immediately.

\section{Case Study}

This research was implemented on a modular factory in Edmonton, $\mathrm{AB}$, Canada, that prefabricates wood structure residential modules under environmentallycontrolled conditions and transports them to sites. Manufacturing provides opportunity for the company to offer time efficiency, cost effectiveness, and superior quality compared to traditional stick-built construction. All houses are built to building code for the given region, and are transported and installed in urban, rural, or remote areas. This factory fabricates customdesigned homes that vary in features, layout, and size between $600 \mathrm{sq} \mathrm{ft}$ and 1,600 sq ft.

\subsection{Simulation of Production Process}

In this phase, simulation models of the current and future states of the production process are generated in Simphony.NET 4.0. The current-state production process is generated based on the factory current-state
VSM. Numbers of labor personnel are constant variables and activity durations are defined by data distributions. The future-state production process is generated based on set of proposed recommendations. The simulation model depicts the production line layout; individual and overall production schedules through the production line for 10 modules that vary in size and specifications; resource requirements based on each module's dimensional properties; and the optimum Takt time to reach an optimum resource allocation plan. The inputs for this model are frontloaded from information in the BIM-generated 3D model of sample modules, which is extracted and sorted into a spreadsheet. Then man-hours requirements are calculated based on modules specification. For the case-study, similar to with on-site construction, a customer can choose from among existing floor plans or provide their own customized floor plan which accommodates their needs and lot size. As a result, the factory production line cannot be run at a steady pace, since the activities taking place at each station are contingent upon individual design. Therefore required man-hours at different workstations are calculated based on modules dimension and specification, which is beyond the scope of this paper. Then required man-hours are imported into a database which is linked to the simulation model. The simulation model delivers results for different production scenarios and provides the opportunity to evaluate the proposed future-state in order to optimize the production process.

\subsubsection{Current-State map}

The current-state simulation model of the factory production line is shown in Figure 2. All activities and their sequences in each station are generated and proper data distributions for the processing time of each activity are defined. In this model, the current-state of the production process is simulated based on the factory current-state VSM for 10 sample modules. The numbers of assigned labor personnel are fixed at each station and there is no cross-training through the production line. Modules vary slightly in size and specifications, entailing that processing times for modules are not uniform. The variation range is limited in order to identify defects in situations in which the source of deficiency is not obvious. The results of the simulation model comprise processing time for sample modules to be fabricated at each station, total processing time, idle time, and total man-hour requirements for the currentstate production process. Variations in processing time at each station for different modules are plotted in output charts as well as total processing times for all the sample modules as presented in Figure 3. The duration variation of processing hours to complete each module at different stations and overall are presented in Figure 4. 


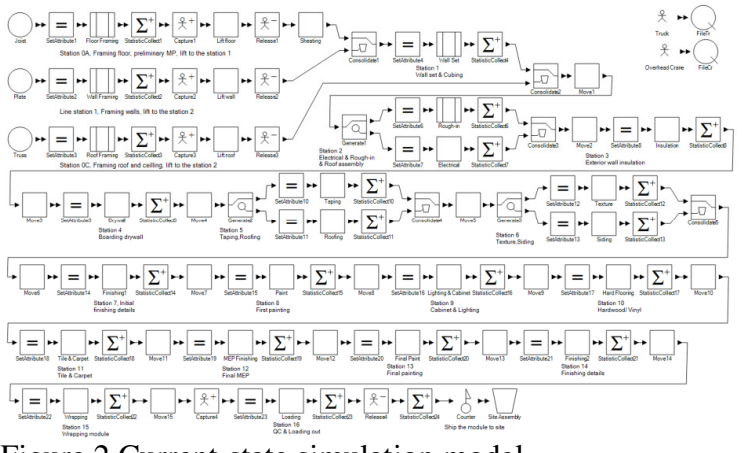

Figure 2 Current-state simulation model

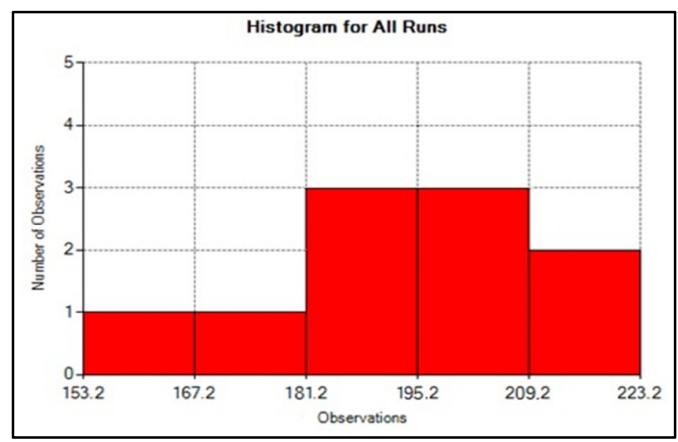

Figure 3 Total processing hours for sample modules in the current-state

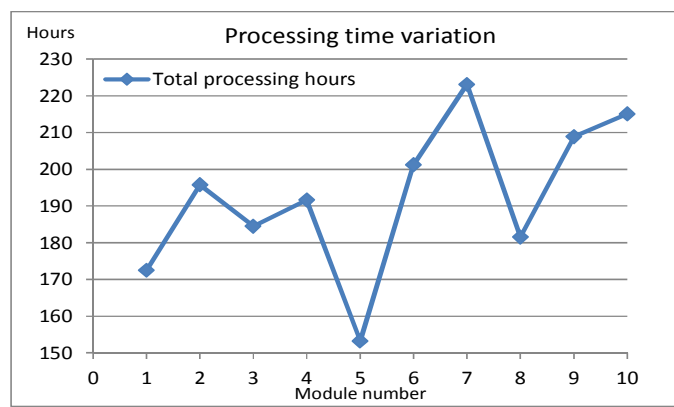

Figure 4 Processing time variation for sample modules in current-state

The results of the simulation model demonstrate the variation in module completion duration at each station. When a larger module enters the production line, it is returned to the bottleneck of the production line, keeping upstream stations idle. Also, modules in the downstream stations are unable to move since the work on the previous module is not complete. As a result, the production capacity is decreased and the scheduled target based on customer demand cannot be reached.

\subsubsection{Future-State map}

The future-state of the production process is generated based on a set of proposed recommendations as follows:

- The number of labor personnel at each workstation is not fixed. Takt time is defined in such a way as to move the line at a steady space and create continuous flow. Different scenarios are therefore run in order to find the near optimum result for the Takt time at which an optimum resource allocation plan is reached with the least fluctuation in labor requirements for different modules.

- The layouts of the off-line framing stations (floor framing, roof/ceiling framing, and wall framing) and cubing station are changed. The location of floor framing station is changed, and some activities are moved upstream including sheathing the floor and marking the walls layout. The proposed layout eliminates three waste activities from the process: lifting the floor with the overhead crane; undoing the air-jacks before lifting and replacing them afterwards; and carrying necessary tools and material to the next station to complete sheathing.

- The process was improved through utilization of more advanced tools and equipment. The required time to manually square the walls is eliminated by means of jigs at the wall framing table. The required time to cut wall components is reduced to half by means of a radial arm saw with measuring ability to cut several pieces to size at once. The required time to prepare a platform for roof/ceiling is reduced by $60 \%$ by means of an adjustable work platform to set up the roof for various layouts.

- $\quad$ The idle time associated with material delivery delay is eliminated through the use of just-in-time delivery along with Kanban cards for material replenishment and handling.

The future-state simulation model, as shown in Figure 5, determines resource allocation plan scenarios of the future-state production process. This model runs the simulation for a series of Takt times and calculates man-hour requirements for a number of sample modules at all the stations through the production line. Then, the best match for number of labor personnel at each station is defined, and, based on this the man-hour fluctuation caused by module variation at individual stations is measured. The model then calculates for each station and for the entire production line (1) total labor idle time due to earlier completion of a module; and (2) additional man-hour requirements due to late completion of a module. The total required time that is covered by idle labors defines the required number of labor personnel in the multi-skill worker crew that is cross-trained through the production line to increase production rate at stations which are behind the scheduled Takt time. The model is run to find the scenario with the minimum man-hours not covered by 
the multi-skill worker crew. Table 1 presents the required number of labor personnel and labor fluctuations at the floor station for sample modules for different scenarios, with Takt times ranging from 6 to 11 hours.

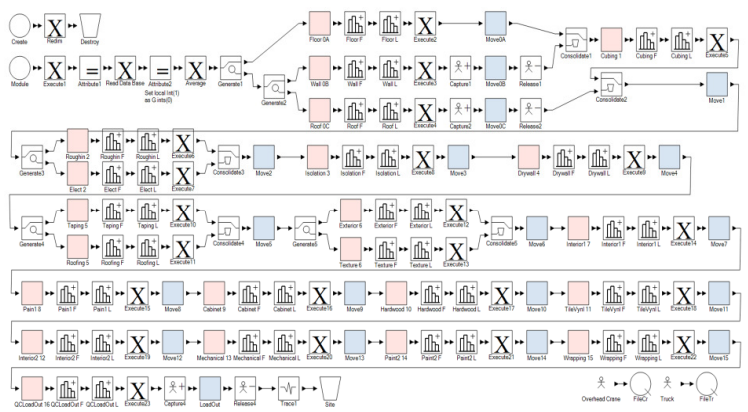

Figure 5 Future-state VSM simulation model

Table 1 Labor requirements and labor fluctuation at floor station for different scenarios

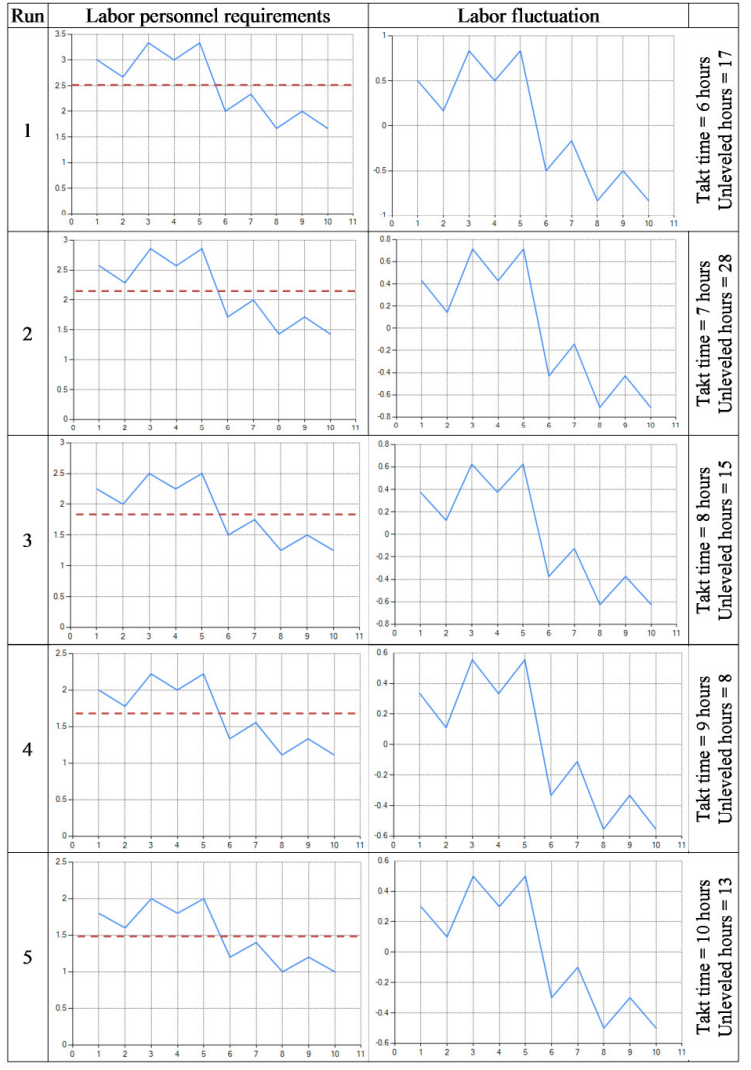

A number of scenarios offered by the simulation model are presented in Table 2. Based on a selected Takt time, which varies between 6 and 11 hours, the number of fixed labor personnel and multi-skill labors change. The results provide various options from which to select according to company strategies. For example, in scenario 1 with 6-hour Takt time, 71 labor personnel are required in total, including 67 stationary labor personnel and 4 multi-skill labor personnel, whereas in scenario 6 with 11-hour Takt time, 37 labor personnel are required in total, all of which are stationary. Although the total number of labor personnel required in scenario 6 is half of that required in scenario 1 , due to the long Takt time the production rate is 21 modules per month, whereas the production rate in scenario 1 is 40 modules per month. A moderate scenario (scenario 3 ) is presented in which the total number of labor personnel is balanced with production rate. A decision on resource allocation can therefore be made by the management team based on the strategic vision of the company.

Table 2 Scenario analysis for future-state production

\begin{tabular}{|c|c|c|c|c|c|c|c|c|}
\hline \multicolumn{9}{|c|}{ process } \\
\hline 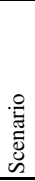 & 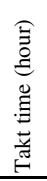 & 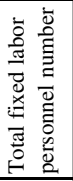 & 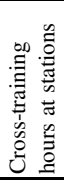 & 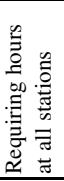 & 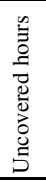 & 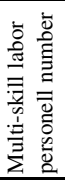 & 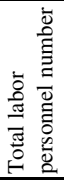 & 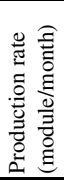 \\
\hline 1 & 6 & 67 & 78 & 105 & 17 & 4 & 71 & 40 \\
\hline 2 & 7 & 56 & 63 & 91 & 28 & 3 & 59 & 34 \\
\hline 3 & 8 & 50 & 60 & 75 & 15 & 2 & 52 & 30 \\
\hline 4 & 9 & 45 & 58 & 66 & 8 & 1 & 46 & 27 \\
\hline 5 & 10 & 40 & 54 & 67 & 13 & 1 & 41 & 24 \\
\hline 6 & 11 & 37 & 54 & 59 & 5 & 0 & 37 & 21 \\
\hline
\end{tabular}

\subsection{Post-Simulation Visualization Production Process}

In this phase, a post-simulation visualization model of the evaluated production scenarios is developed which facilitates production flow analysis and decision making for the management team. This model visualizes the results of the simulation model and provides the opportunity to compare near-optimum production scenarios, not only through charts and statistical data, but also by means of visualizing the ideal process prior to actual implementation of the proposed changes.

\subsubsection{Animation and Process Evaluation}

The proposed method integrates 3D visualization of a process with the statistical results of the simulation. The generated post-simulation visualization (PSV) model visualizes the simulation of the production process in detail, presenting process information such as modules' fabrication schedule (both individually and in total) throughout the production line for evaluation purposes, whereas the proposed technique has the capacity to be linked to all statistical production information. For this purpose, a general visual 
representation of the production process is developed in Autodesk 3D Studio Max (3ds Max), showing certain activities at all stations in the production line. After evaluating and comparing simulation results between various production scenarios, a scenario with a near optimum result is selected to be visualized. The processing times of all the stations are imported from the simulation model output and linked to the 3D animation semi-automatically. A snapshot of the generated PSV model is presented in Figure 6.

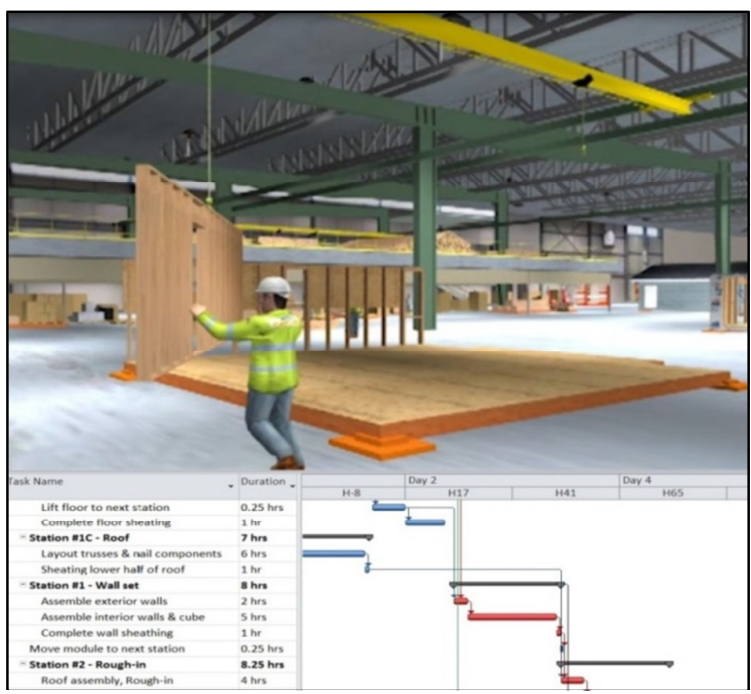

Figure 6 Snapshot of PSV model developed in 3ds Max

The visualization of the model also provides opportunity for further analysis, such as the following tasks: (1) overhead crane examination as presented in Figure 7(a) for proper lifting at wall station to find optimum distance between framing table and cubing station, and at roof station to find lifting points for roof and ceiling; (2) $5 \mathrm{~S}$ implementation as presented in Figure 7(b) to develop proper layout and space in order to achieve the 5S goals; (3) review of ergonomic and safety issues arising throughout the process, as presented in Figure 7(c), and proposing of a solution; (4) visualization of any required changes prior to implementation, as illustrated in Figure 7(d); this task includes both presenting the module features, specifications, and appearance to customers, and also presenting the proposed factory layout configuration to the management team, such as the improved layout for the warehouse area; and (5) evaluation of truck capacity considering module size in order to ensure proper transportation of modules, and selection of suitable trucks based on module width and weight, as displayed in Figure 7(e).

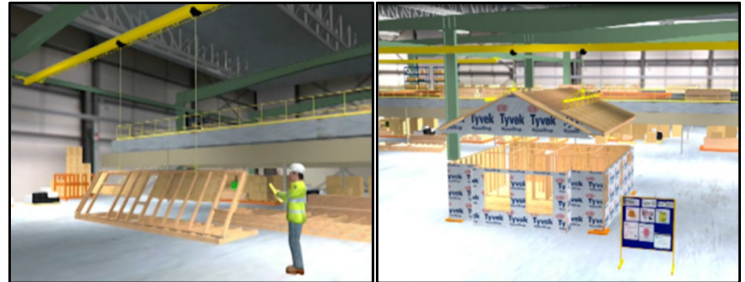

(a) Examination of overhead crane for proper lifting

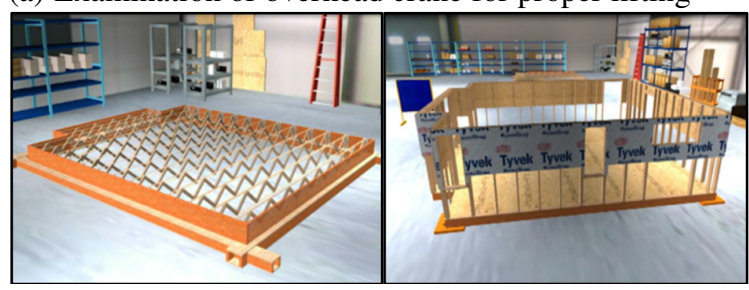

(b) $5 \mathrm{~S}$ implementation and evaluation of proper layout

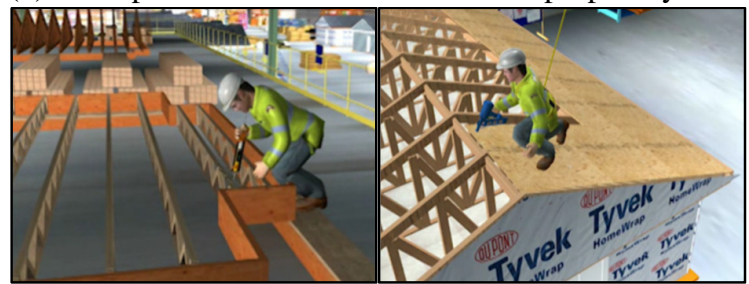

(c) Ergonomic and safety issues

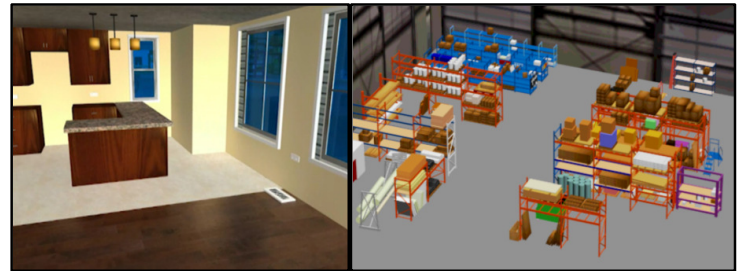

(d) Module and factory presentation

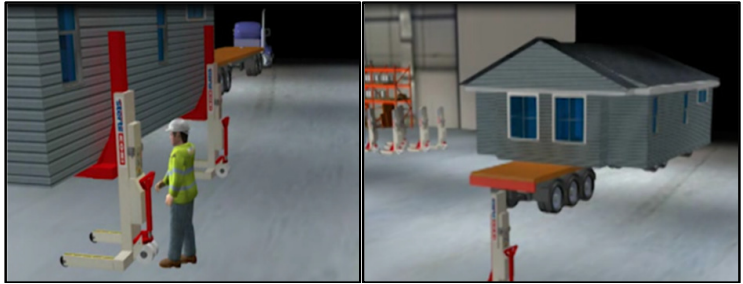

(e) Truck capacity analysis to load-out modules

Figure 7 Further analysis by means of PSV model

\subsubsection{Animation and Process Evaluation}

A complex module production process which includes various constraints such as time, predecessor activity networks, space, continuous flow, labor assignments, and product variation, requires a flexible evaluation model to deal with changes in process and present the future-state in a simple way. For this purpose, another approach is pursued to create a more dynamic visualized model of the simulation results in Autodesk Navisworks Manage. The 3D model of the 
factory is developed in Revit and then, along with the $3 \mathrm{D}$ models of modules generated in the design phase, is imported into Navisworks. Required information pertaining to modules currently in the production line and coming modules, including the processing times at all the stations, resource allocation plan, and work sequence, are imported from the simulation output for different scenarios. In order to analyze different plant layout configuration scenarios with automatic clash detection, more information is added to the model, including overhead crane capacity, factory space limitations, and labor safe work area.

In this model, a minute-by-minute schedule of the production process is generated and various layout configurations are analyzed for optimum scenarios. The developed PSV model capitalizes both on the advantages of the simulation as well as on the simplicity of the visualized minute-by-minute schedule. Figure 8 shows a snapshot from the model's output for the case study that presents the process for scenario 3 with 8hour Takt time. The model reveals a number of clashes due to space limitation for module transportation. In order to reach the production capacity, the number of produced modules is not changed, but the layout of the workstation arrangement changes until no clash is detected. Furthermore, an investigation is carried out considering various production process scenarios in order to compare the resource allocation plans and identify the best potential scenario for the given number of labor personnel at each station. The result of comparing the initial simulation model before generating PSV and the final version after running PSV indicates significant improvements in the production schedule and resource allocation.

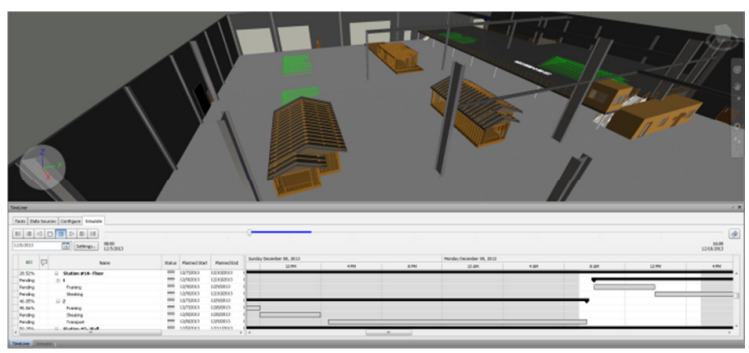

Figure 8 PSV model of scenario 3 with 8-hour Takt time

\section{Conclusion}

With the capacity to predict and evaluate different scenarios for the production line, the introduction of simulation tools to resource planning provides several advantages to user in reducing risk of failure, accurate production forecast, and Takt time prediction. Since results are based on statistical information and specific expertise on simulation, PSV models provide a better understanding of simulation outcomes for managers and workers. However, the two-presented visualization models have different purposes and scopes. The first model presents in detail the planned manufacturing process after decisions being made from simulation result. It has, therefore, a more reviewing purpose in order to demonstrate how the process is carried out. The second model is used as a tool to visualize the effect of the activity network in the schedule and predict clashes that the simulation model is not able to detect as the conflict with models and the space limitation of facility.

\section{References}

[1] Detty, R. B. and Yingling, J. C. Quantifying benefits of conversion to Lean manufacturing with discrete event simulation: A case study. International Journal of Production Research, 38(2), 429-445, 2000.

[2] Moghadam, M., Al-Hussein, M., Al-Jibouri, S., and Telyas, A. Post simulation visualization for effective scheduling of modular building construction. Canadian Journal of Civil Engineering, 39(9), 1053-1061, 2012 b.

[3] Shararah, M. A., El-Kilany, K. S., and El-Sayed, A. E. Value Stream Map simulator using ExtendSim. Proceedings, World Congress on Engineering, Vol. I, London, UK, Jul. 6-8, 755-758, 2011.

[4] Marvel, J. H. and Standridge, C. R. A simulationenhanced Lean design process. Journal of Industrial Engineering and Management, 2(1), 90113, 2009.

[5] Al-Hussein, M., Niaz, M. A., Yu, H., and Kim, H. Integrating $3 \mathrm{D}$ visualization and simulation for tower crane operations on construction sites. Automation in Construction, 15(2), 554-562, 2006.

[6] Kamat, V. R., and Martinez, J. C. 3D visualization of simulated construction operations. Winter Simulation Conference, Orlando, FL, USA, vol. 2, 1933-1937, 2000.

[7] Zhong, Y., Shirinzadeh, B., Ma, W. Solid modelling in a virtual reality environment. The Visual Computer, 2005.

[8] Telea, A. C. Visualization and simulation with object-oriented networks. Ph.D. thesis, Eindhoven University of Technology, Eindhoven, The Netherlands, 2000.

[9] AbouRizk, S. M. and Hajjar, D. A framework for applying simulation in construction. Canadian Journal of Civil Engineering, 25(3), 604-617, 1998.

[10] Hajjar, D. and AbouRizk, S. M. Unified modeling methodology for construction simulation. Journal of Construction Engineering and Management, 128(2), 174-185, 2002. 